\title{
CONTEXT-SENSITIVE REFERENCE FIXING AND OBJECTIVITY. REPLY TO BARCELÓ 1
}

\author{
MARIO GÓMEZ-TORRENTE \\ https:/ / orcid.org/0000-0002-5957-6544 \\ Instituto de Investigaciones Filosóficas \\ Universidad Nacional Autónoma de México (UNAM) \\ Mexico City \\ Mexico \\ mariogt@unam.mx
}

\begin{abstract}
Article info
CDD: 128.68

\section{Keywords}

Color Objectivism

Context Sensitivity

Aesthetic Predicates

Reference Fixing
\end{abstract}

Received: 01.10.2020; Accepted: 17.10.2020

https://doi.org/10.1590/0100-6045.2020.V43N4.MB

Abstract: Axel Barceló has extended the objectivist apparatus for handling color terms that I develop in my book Roads to Reference, so that the extension covers also some aesthetic predicates. In this note I argue that Barceló's extension probably attempts to go too far.

Axel Barceló (2020) seeks to give a general version of my account of reference fixing for color and temperature adjectival expressions (in Roads to Reference and in GómezTorrente (2016); see also additions in Gómez-Torrente

${ }^{1}$ Support from the research project no. PIDPID-107667GB-I00 of the Spanish Ministry of Science and Innovation is gratefully acknowledged. 
(2017)), one that can apply to many other context-sensitive expressions. Under my account, particular uses of, say, the adjective "green", refer not to a subjective property such as looking green to Mario (in normal conditions), but to an objective property such as irradiating light between levels $a$ and $b$ in the objective dimension of bue (in normal conditions), where $a$ and $b$ are hue levels of green somehow taken as standards by my (typically implicit) contextual intentions. Barceló generalizes the idea that the referent of a context-sensitive expression may be objective even if the aspects of context that help fix it involve subjective elements. I sympathize with the project of generalizing the account, and I agree with Barceló on some of the expressions that a generalized account should apply to, but I see problems with the way in which he makes the generalization, and with some of the examples of expressions that he wants the generalization to apply to.

Barcelós generalization adopts the form of a test designed to determine when certain context-sensitive expressions get objective referents-the "determinate values of determinable property $\mathrm{P}$ " in what follows:

Whenever there are predicates P1 and P2 (and sometimes P3, P4, etc.) such that there is a (determinable) property $\mathrm{P}$, at least one object $\mathrm{X}$ and a pair of contexts $\mathrm{C} 1$ and $\mathrm{C} 2$ such that: Part I: Sensibility. 1. In context C1, a (literal, assertoric, non-descriptive, de re, etc.) utterance of " $\mathrm{X}$ is $\mathrm{P} 1$ " is true. 2. In context $\mathrm{C} 2$, a (literal, assertoric, non-descriptive, de re, etc.) utterance of "X is P2" is true. 3. P1 and $\mathrm{P} 2$ are incompatible predicates (nothing can be both P1 and P2 at the same time). Part II: Stability. 4. Both " $\mathrm{X}$ is $\mathrm{P} 1$ " and " $\mathrm{X}$ is $\mathrm{P} 2$ " are adequate answers to the question "How $\mathrm{P}$ is $\mathrm{X}$ ?" (or similar: "What is the $\mathrm{P}$ of $\mathrm{X}$ ?", 


\begin{abstract}
"Which P is X?", “How does X P?”, etc. given that $\mathrm{P}$ is a determinable property). 5. Yet, changing $\mathrm{X}$ from $\mathrm{C} 1$ to $\mathrm{C} 2$ does not change how $\mathrm{P} \mathrm{X}$ is. Then, $\mathrm{P} 1$ and $\mathrm{P} 2$ (which are sensitive to contextual differences between $\mathrm{C} 1$ and C2) refer to (determinate values of determinable) property $\mathrm{P}$ (which is not sensitive to those contextual differences).
\end{abstract}

In order to try to understand this, let's see how it might apply in an example similar to the ones used in Roads to Reference, involving the two chromatic adjectival expressions "pure green" and "bluish green", which are incompatible (as in 3.): There is a colored chip $\chi$ such that (1.) Jones utters, apparently faultlessly, $\chi$ is pure green, while (2.) Smith utters $\chi$ is bluish green, also apparently faultlessly. (Roads to Reference postulates that each of the two utterances is made in a different context, as Jones intends certain standards in the dimension of hue relevant to the gradability of "green" which are different from the standards intended by Smith.) (4.) Both $\chi$ is pure green and $\chi$ is bluish green are adequate answers to questions such as How green is $\chi$ ? or What color is $\chi$ ? Now as to (5.), does "changing $\chi$ from the context of Jones' utterance to the context of Smith's" change how green $\chi$ is, or what color $\chi$ is"? Obviously Barceló intends the question to be a clear "No", presumably because he thinks that $\chi$ doesn't change color merely by "changing context". However, I'm a bit unclear about what it means to "change an object from one context to another", and consequently about what it means to ask whether an object can change color from context to context. I think the notion is a bit confusing, and as its clarity stands, I don't see why one might not think that when $\chi$ is considered in Jones' context, it is pure green, while when $\chi$ is considered 
in Smith's context, it is bluish green, the answer to the (5.) question thus being "Yes, change of context may imply change of color".

It may be worth noting that when I speak of "modal stability" in Roads to Reference (see Gómez-Torrente (2019), 192), I mean something different from, and, I think, less confusing than what Barceló means by "stability". A predicate Q is modally stable just in case, for any object o, if Q applies to o in a certain context $\mathrm{c}$ and world $\mathrm{w}$, then it applies to $\mathrm{o}$ in the same context $c$ in any world w' where o has the same objective properties as in w. In other words, if the context is kept fixed and the world of evaluation is changed to a world where o doesn't change as to its objective properties, then Q keeps applying to o-that's what makes Q modally stable. Intuitively, color predicates are modally stable, because an object doesn't change color if its objective properties don't change and we are considering it from the same context (where a context is thought of as involving a particular set of standards, i.e. levels in the dimensions of hue, saturation and brightness). There is no question here as to whether an object may change color when we consider it from a context different from the one originally considered. On a natural way of seeing the matter, embraced in Roads to Reference, the answer to that question may well be "Yes". But the test that seems important for the question as to whether color predicates have objective referents is the test of modal stability, and here the answer is an intuitively clear "No": $\chi$, say, doesn't change color from world to world as long as its objective properties don't change.

Barceló wants to apply his generalized schema to "tasty" and "disgusting", taken as antonyms. He says:

There seem to be contexts C1 and C2 where

1. In context $\mathrm{C} 1$, a (literal, assertoric, non- 
descriptive, de re, etc.) utterance of " $\mathrm{X}$ is tasty" is true. 2. In context C2, a (literal, assertoric, non-descriptive, de re, etc.) utterance of " $\mathrm{X}$ is disgusting" is true. 3. Tasty and disgusting are incompatible predicates (nothing can be both tasty and disgusting at the same time)... 4. Both " $\mathrm{X}$ is tasty" and " $\mathrm{X}$ is disgusting" are adequate answers to the question "How does X taste?". 5. Yet changing $\mathrm{X}$ from $\mathrm{C} 1$ to $\mathrm{C} 2$ does not change how $\mathrm{X}$ tastes.

Well, nothing to object to 1 . through 4., but I'm again unclear about 5. What does it mean exactly to "change $\mathrm{X}$ from C1 to C2"? Barceló says that "to say that "tasty" and "disgusting" are context sensitive is nothing but the antirealist intuition that the same stuff can be tasty to some, but not to others", so I suppose that what may change from context to context is the taster, and with her the criteria for whether X counts as tasty or not. But then, as before, why can't we say that how X tastes changes from C1 to C2?

Barceló here adds something that might be potentially clarifying:

stability [essentially 5 , that is] is nothing but the intuition that, in absence of conflicting top-down expectations, whenever one finds something tasty, if, instead of that thing, one had tasted something else that tasted the same, one would have found it tasty as well.

But I find this more puzzling than illuminating: considering how one same taster reacts to two different but molecularly identical bits of food is certainly not the same as considering what happens when you make two different 
tasters react to the same bit of food. I surely agree that "tasty" and "disgusting" are stable if this just means that the same taster reacts in the same way to molecularly identical bits of food; but this is not the same as to say that the same bit of food doesn't change taste from taster to taster-and to the extent that I understand this, I would say it does change taste.

What happens if we apply the test of modal stability? "Tasty" (analogous points will hold for "disgusting") is modally stable just in case, for any object o, if "tasty" applies to $\mathrm{o}$ in a certain context $\mathrm{c}$ and world $\mathrm{w}$, then it applies to $\mathrm{o}$ in the same context $\mathrm{c}$ in any world w' where $\mathrm{o}$ has the same objective properties as in w. We can assume that in this case this implies that if the taster is kept fixed and the world of evaluation is changed to a world where $\mathrm{X}$ doesn't change as to its objective properties, then "tasty" keeps applying to X. If this is all, then intuitively "tasty" is not modally stable, because the taster may be the same in w' and yet have changed so much (physiologically, for example) that $\mathrm{X}$ (which has not changed) does no longer count as tasty for her. Now it might be said (though Barceló doesn't say this, as far as I can see) that in the case of "tasty" the relevant aspect of the context is not defined merely by the taster, but (as in the case of "green") by appropriate contextual standards fixed by tasters along a certain dimension of tastiness, and that as long as these don't change, if the world of evaluation is changed to a world where X doesn't change as to its objective properties, then "tasty" will keep applying to X. But in order for this to sound plausible, we would have to have a well identified (objective) dimension of tastiness comparable to the dimensions of hue, saturation and brightness in the case of "green". And I think most people would say that they can't see what that objective dimension would be. 
Barceló says things that might lead one to think that he believes such a dimension exists. With 5 . above in the application to "tasty" and "disgusting", for example, he may mean that, regardless of what taster is involved, how X tastes (as long as it does not change its molecular structure) is something that doesn't change. Now I would perhaps be ready to accept that in some sense X's objective taste or flavor doesn't change if its molecular structure doesn't change. But I think that a quite different matter is whether the adjectives "tasty" and "disgusting", taken as antonyms, have semantic application conditions that involve an objective dimension along which standards are imposed by context. I can't see any evidence for this, and in fact I think it's evident that both "disgusting" and "tasty" taken as antonyms are as evaluative and subjective as anything can possibly be, and there is no objective dimension that ranks things as to their level of "disgustingness".

Consider also "handsome" (with "ugly" as an antonym?), another example to which Barceló wants to apply his generalization but which I find suspicious. He says that "it seems to be as stable as well, because for any handsome subject, anybody that looks the same would also be considered handsome" (note again how this seems different from saying that the same person will not change as to "handsomeness" from aesthetic judge to aesthetic judge-assuming this is the relevant aspect of context here). Is this really how we want to test for the objectivity of the property involved? Suppose we stipulate that a new word, "schmhandsome", is to have a subjectivist semantics-that, as used by a speaker S on a particular occasion, "schmhandsome" refers to to the property of being physically liked by $\mathrm{S}$ (and analogously for "schmugly"). According to Barceló's stability test, "schmandsome" presumably comes out stable, because for any schmhandsome subject, anybody that looks the same 
will also be considered schmhandsome (by the same speaker on the same occasion). I think that if even words that we explicitly stipulate to have a subjective semantics pass Barceló's test for objective reference, the test cannot be good. ${ }^{2}$

By contrast, I think that Barceló's suggestion that "salty" (and "insipid" as an antonym, say), in its predominant acceptation, has an objectivist semantics is on the right track (though I'm skeptical that this is for the reasons he has in mind). There is certainly an objective dimension that ranks things, in particular bits of food, as to their saltiness, namely the dimension of amounts of salt contained in a bit. And intuitively one can adopt different contextual standards along this dimension: the same amount of salt may make someone say of a bit of food that it's salty and someone else say that it's not salty, or even insipid, presumably because they intend different standards in the dimension of amounts of salt (for that bit, or for bits of a similar composition) as determining the application or otherwise of "salty" (and "insipid"). Not surprisingly, "salty" (and "insipid") intuitively pass the modal stability test: for any bit o, if "salty" ("insipid") applies to o given a certain standard s (in the dimension of amounts of salt) in a world $\mathrm{w}$, then intuitively it applies to o given the same standard s in any world w' where o has the same objective properties as in $\mathrm{w}$-including of course, the amount of salt in 0.

2 The reader may have recognized here an application of Kripke's (1977) useful test involving imaginary stipulated languages.

Manuscrito - Rev. Int. Fil. Campinas, v. 43, n. 4, pp. 13-21, Oct.-Dec. 2020. 


\section{REFERENCES}

Barceló, A. (2020), "Tasty Roads to Flavour", Manuscrito, this issue.

Gómez-Torrente, M. (2016), "Perceptual Variation, Color Language, and Reference Fixing. An Objectivist Account", Noûs 50, 3-40.

Gómez-Torrente, M. (2017), "Objectivism about Color and Comparative Color Statements: Reply to Hansen", Noûs 51, 429-35.

Gómez-Torrente, M. (2019), Roads to Reference. An Essay on Reference Fixing in Natural Language, Oxford University Press, Oxford.

Kripke, S. A. (1977), "Speaker's Reference and Semantic Reference", Midwest Studies in Philosophy 2, 255-76.

$(c)) \mathrm{BY}$ 